\title{
Non-Linear Beam Formulation with NURBS Interpolation for the Simulation of Sliding Contacts
}

\author{
Paul Wasmer, Peter Betsch \\ Institute of Mechanics \\ Karlsruhe Institute of Technology (KIT) \\ Otto-Ammann-Platz 9, 76131 Karlsruhe, Germany \\ paul.wasmer@kit.edu, peter.betsch@kit.edu
}

\begin{abstract}
The simulation of beams with sliding joints is of interest for many different applications such as aerial runways or pantograph-catenary systems. However, classical discretization with Lagrangian finite elements cannot represent the geometry smoothly over element boundaries, which leads to impacts at element boundaries. Therefore, the isogeometric framework is applied to a director based formulation of the geometrically exact beam formulation using non-rational B-Splines (NURBS) for the discretization as NURBS can be used to represent many geometries smoothly and exactly. An energy-momentum scheme is used for the time integration to obtain a stable algorithm. Two methods are proposed to realize the sliding contact condition: a classical approach using Lagrange multipliers and an approach based on the masterslave concept applied in the framework of the discrete null space method.
\end{abstract}

Keywords: geometrically exact beams, sliding contact, null space method, energymomentum schemes

\section{Introduction}

The simulation of sliding contacts of two (or more) slender structures is of interest for many different applications such as aerial runways or pantograph-catenary systems. Slender structures can be simulated very efficiently using beam models. One of the most important beam models in the non-linear analysis is the geometrically exact beam formulation (or Reissner-Simo beam theory) $[1,2,3]$. The geometrically exact beam formulation allows arbitrarily large deformations with finite strains, while the cross-section of the beam remains planar. The orientation of the beam's cross-section is often described using rotational variables. However, without additional effort, the discretization of the rotations with finite elements is not frame-indifferent [4]. Thus, we use a formulation based on directors, three orthonormal vectors, which describe the orientation of the beam's cross-section $[5,6,7,8]$.

Hamiltonian mechanical systems, such as beams, are based on the balance laws for linear and angular momentum as well as the balance of energy in case of conservative loadings. However, these conservation properties might not carry over to the discretized time domain. Especially, in the case of nonlinear configuration manifolds, this might lead to physical impossible solutions or numerical instabilities [9]. A remedy for this problem is the use of conserving integrators, which preserve some of the structure of the continuous problem. A general approach on how to construct such energy-momentum conserving integrators was shown by Gonzalez [9]. The conserving time integration scheme, which preserves momentum and energy, for elastodynamics was first proposed in [10]. In [11] Simo et. al showed the advantage of the energy-momentum scheme over the conventional midpoint and trapezoidal rule when used for the geometrically exact beam. Therefore, the energy-momentum scheme is applied to simulate the dynamic behavior of the beam.

For the simulation of a sliding contact, it is essential to represent the geometry without any nonphysical kinks, which may arise due to the discretization. Otherwise, this leads to impacts at the 
kinks, which are not present in the continuous problem. A smooth representation of the geometry is not possible if the classical isoparametric finite element method (FEM) in combination with Lagrangian shape functions is used, as here no smooth representation over element boundaries is possible. The isogeometric analysis (IGA) [12] can present a remedy for this problem. The IGA can be used for an exact, smooth representation of the whole geometry. In the IGA framework the functions, which are used to exactly display the geometry in computer graphics, are also used as basis functions for the FEM discretization. Often non-uniform rational B-Splines (NURBS) are applied for this purpose. NURBS are $C^{p-1}$ continues over element boundaries, where $p$ is the polynomial order of the shape functions.

It is the goal of the present work to achieve a contact algorithm, which adopts the conserving property of the energy-momentum scheme. For this purpose, two methods to enforce the contact condition are investigated: First, the more classical approach using Lagrange multipliers is stated. The second approach relies on the master-slave concept $[13,14,15,16]$ which is applied within the discrete null space method $[17,18,19]$.

An outline of the rest of the paper is as follows. In Section 2 we give a very short summary on the used NURBS basis functions. It follows a detailed description of the geometrically beam formulation relying on directors in Section 3, along with an outline of the applied energy-momentum scheme. The sliding contact condition is addressed in Section 4. Eventually, some conclusions are drawn in Section 5.

\section{Isogeometric Analysis}

First introduced by Hughes et al. [20] in 2005, the IGA using NURBS was applied to a variety of problems since then and has become it's own field of research. The origin of NURBS basis functions lies in computer graphics. A comprehensive introduction to the topic of NURBS can be found in [21]. A NURBS curve is built from B-Splines. Each B-Spline function is defined through a knot vector

$$
\Xi_{p}=[\underbrace{0, \ldots 0}_{p+1}, \underbrace{\xi_{p+2}, \ldots, \xi_{n_{\mathrm{ele}}+p+1}}_{n_{\mathrm{ele}}-1}, \underbrace{1, \ldots 1}_{p+1}]
$$

where the index $p$ denotes the polynomial degree of the shape function and $n_{\text {ele }}$ the number of curve segments, which is equivalent to the number of elements in a FEM sense. Eq. (1) defines an open knot vector, which is classically used in IGA. An open knot vector defines functions, where the basis functions are interpolatory at the beginning $(\xi=0)$ and the end $(\xi=1)$. Using the knot vector a B-Spline basis can be computed with the help of the Cox-de-Boor recursive algorithm $[21,12]$

$$
\begin{aligned}
& N_{i, 0}(\xi)= \begin{cases}1 & \text { if } \xi_{i} \leq \xi<\xi_{i+1} \\
0 & \text { otherwise }\end{cases} \\
& N_{i, p}(\xi)=\frac{\xi-\xi_{i}}{\xi_{i+p}-\xi_{i}} N_{i, p-1}(\xi)+\frac{\xi_{i+p+1}-\xi}{\xi_{i+p+1}-\xi_{i+1}} N_{i+1, p-1}(\xi)
\end{aligned}
$$

where division by zero is defined as zero $\left(\frac{\bullet}{0}:=0\right)$. The NURBS basis is constructed form

$$
R_{i}^{p}(\xi)=\frac{N_{i, p}(\xi) w_{i}}{W(\xi)}=\frac{N_{i, p}(\xi) w_{i}}{\sum_{\hat{i}}^{n \text { CP }} N_{\hat{i}, p}(\xi) w_{\hat{i}}}
$$

where $w_{i}$ is the $i$-th weight. If all weights are set to one $\left(w_{i}=1\right)$ the NURBS basis coincides with the B-Spline basis defined above. With the control points $\mathbf{B}_{i}$, where $\mathbf{B}_{i} \in \mathbb{R}^{d}$ is the $i$-th control point, a NURBS curve can be constructed

$$
\mathbf{C}(\xi)=\sum_{i=1}^{n_{\mathrm{CP}}} R_{i}^{p}(\xi) \mathbf{B}_{i}
$$


where $n_{\mathrm{CP}}=n_{\text {ele }}+p$ is the number of control points. NURBS curves can be used to display many common geometries exactly and smoothly.

\section{Geometrically Exact Beam Formulation}

Many different formulations for non-linear beams exist in the literature. The here presented formulation follows closely the following publications $[5,6,7,8]$.

\subsection{Beam Kinematics}

The position of any point on the beam can be described by

$$
\mathbf{x}\left(s, t, \theta^{1}, \theta^{2}\right)=\boldsymbol{\varphi}(s, t)+\boldsymbol{\theta}(s, t)=\boldsymbol{\varphi}(s, t)+\theta^{\alpha} \mathbf{d}_{\alpha}(s, t)
$$

where $s \in\left[s_{1}, s_{2}\right]$, with $s_{1}, s_{2} \in \mathbb{R}$, is referred to as the arc-length in the reference configuration. $\theta^{i} \in \mathbb{R}$ are convective coordinates $\left(\theta^{1}, \theta^{2}, \theta^{3}=s\right) . \varphi \in \mathbb{R}^{3}$ points to the centerline of the beam and $\theta^{\alpha}$ gives the position on the cross-section. The Einstein notation for double indices is used. Note that from now on indices with Greek letters run from one to two $(\alpha, \beta=1,2)$, whereas indices with Roman letters run from one to three $(i, j, k=1,2,3)$.

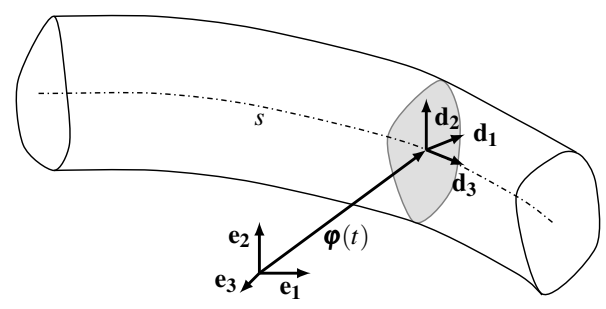

Figure 1. Configuration of the beam

Three directors $\mathbf{d}_{i} \in \mathbb{R}^{3}$ are defined, where $\mathbf{d}_{1}$ and $\mathbf{d}_{2}$ span the cross-section area of the beam, and $\mathbf{d}_{3}$ is defined by

$$
\mathbf{d}_{3}(s, t)=\mathbf{d}_{1}(s, t) \times \mathbf{d}_{2}(s, t)
$$

In the reference configuration at time $t=0$ the director $\mathbf{d}_{3}(s, 0)$ is equivalent to the tangent of the centerline $\mathbf{d}_{3}(s, 0)=\boldsymbol{\varphi}, s(s, 0)$. Here the abbreviation of the partial derivative $\frac{\partial(\bullet)}{\partial s}$ is introduced. The directors are mutually orthonormal for all $t \in \mathbb{R}$, that is

$$
\mathbf{d}_{i}(t) \otimes \mathbf{d}_{i}(t)=\mathbf{I}
$$

where $\mathbf{I}$ is the unit tensor. The relation between the orthonormal, Cartesian basis $\mathbf{e}_{i}$ and the directors $\mathbf{d}_{i}$ can be expressed by

$$
\mathbf{d}_{i}=\mathbf{R}(s, t) \cdot \mathbf{e}_{i}
$$

where

$$
\mathbf{R}(s, t)=\mathbf{d}_{i}(s, t) \otimes \mathbf{e}_{i}
$$

$\mathbf{R} \in \mathrm{SO}(3)$ is an orthogonal tensor belonging to the special Euclidean group $\mathrm{SO}(3)$.

\subsection{Constitutive Equations}

We introduce two strain measures $\Gamma$ and $\mathbf{K}$

$$
\begin{aligned}
\Gamma & =\Gamma_{i} \mathbf{e}_{i} \text { with } \Gamma_{i}=\mathbf{d}_{i} \cdot \boldsymbol{\varphi}_{, s}-\delta_{i 3} \\
\mathbf{K} & =K_{i} \mathbf{e}_{i} \text { with } K_{i}=\frac{1}{2} \varepsilon_{i j k}\left[\mathbf{d}_{k} \cdot \mathbf{d}_{j, s}-\left.\left(\mathbf{d}_{k} \cdot \mathbf{d}_{j, s}\right)\right|_{t=0}\right]
\end{aligned}
$$

where $\varepsilon_{i j k}$ is the Levi-Civita symbol. Both strain measures are invariant under rigid body movements, which also carries over to the discretized measures as shown in [5]. We assume a Saint 
Vernant-Kirchhoff type material model, where the stored energy is given by the function $w(\boldsymbol{\Gamma}, \mathbf{K})$. We obtain the constitutive equations from

$$
\overline{\mathbf{N}}=\frac{\partial w(\boldsymbol{\Gamma}, \mathbf{K})}{\partial \boldsymbol{\Gamma}} \quad \text { and } \quad \overline{\mathbf{M}}=\frac{\partial w(\boldsymbol{\Gamma}, \mathbf{K})}{\partial \mathbf{K}}
$$

The total strain energy follows from

$$
W=\frac{1}{2} \int_{0}^{L} \boldsymbol{\Gamma} \cdot \mathbf{D}_{\mathbf{1}} \cdot \boldsymbol{\Gamma}+\mathbf{K} \cdot \mathbf{D}_{\mathbf{2}} \cdot \mathbf{K} \mathrm{d} s
$$

with

$$
\mathbf{D}_{\mathbf{1}}=\left[\begin{array}{ccc}
G A_{1} & 0 & 0 \\
0 & G A_{2} & 0 \\
0 & 0 & E A
\end{array}\right] \mathbf{e}_{i} \otimes \mathbf{e}_{j} \quad \mathbf{D}_{2}=\left[\begin{array}{ccc}
E I_{1} & 0 & 0 \\
0 & E I_{2} & 0 \\
0 & 0 & G J
\end{array}\right] \mathbf{e}_{i} \otimes \mathbf{e}_{j}
$$

\subsection{Kinetic Energy}

The kinetic energy of the beam is given by

$$
\begin{aligned}
E^{\mathrm{kin}} & =\frac{1}{2} \int_{0}^{L} \rho_{0}(\boldsymbol{\theta}, s) \dot{\mathbf{x}}(s, t) \cdot \dot{\mathbf{x}}(s, t) \mathrm{d} V \\
& =\frac{1}{2} \int_{0}^{L}\left[A_{\rho} \dot{\boldsymbol{\varphi}}(s, t) \cdot \dot{\boldsymbol{\varphi}}(s, t)+2 \dot{\boldsymbol{\varphi}}(s, t) \cdot \dot{\mathbf{d}}_{\alpha} S^{\alpha}+M_{\rho}^{\alpha \beta} \dot{\mathbf{d}}_{\alpha} \cdot \dot{\mathbf{d}}_{\beta}\right] \mathrm{d} s
\end{aligned}
$$

where $A_{\rho}(s)$ it the mass density per unit reference length

$$
A_{\rho}(s)=\int_{A_{s}} \rho_{0}(\boldsymbol{\theta}, s) \mathrm{d} A
$$

with the density function $\rho_{0}(s) . A_{s}(s)$ is the beam cross-section at $s$. The first moment of area is computed from

$$
\mathbf{S}_{\rho}(s)=\int_{A_{s}} \rho_{0}(\boldsymbol{\theta}, s) \boldsymbol{\theta} \mathrm{d} A
$$

with the components $S_{\rho}^{\alpha}=\mathbf{S}_{\rho} \cdot \mathbf{e}_{\alpha}$ and the mass-moment of inertia of the cross-section is given by

$$
\mathbf{M}_{\rho}(s)=\int_{A_{s}} \rho_{0}(\boldsymbol{\theta}, s) \boldsymbol{\theta} \otimes \boldsymbol{\theta} \mathrm{d} A
$$

with the components $M_{\rho}^{\alpha \beta}=\mathbf{M}_{\rho}:\left(\mathbf{e}_{\alpha} \otimes \mathbf{e}_{\beta}\right)$

\subsection{Constraints}

Lagrange multipliers $\boldsymbol{\lambda}$ are introduced to enforce the orthonormality of the directors

$$
\boldsymbol{\lambda}(s, t):\left[\mathbf{d}_{i} \otimes \mathbf{d}_{i}-\mathbf{I}\right]=\boldsymbol{\lambda}(s, t): \boldsymbol{\Phi}_{\text {in }}=0
$$

where the constraints $\boldsymbol{\Phi}_{\text {in }}$ are given by

$$
\boldsymbol{\Phi}_{\text {in }}=\mathbf{d}_{i} \otimes \mathbf{d}_{i}-\mathbf{I}
$$

\subsection{Virtual Work}

Using the variations of the variables we obtain the variations of the strain measures as

$$
\begin{aligned}
\delta \boldsymbol{\Gamma}=\delta \Gamma_{i} \mathbf{e}_{i} & \text { with } \delta \Gamma_{i}=\delta \mathbf{d}_{i} \cdot \boldsymbol{\varphi}_{, s}+\mathbf{d}_{i} \cdot \delta \boldsymbol{\varphi}_{, s} \\
\delta \mathbf{K}=\delta K_{i} \mathbf{e}_{i} & \text { with } \delta K_{i}=\frac{1}{2} \varepsilon_{i j k}\left[\delta \mathbf{d}_{k} \cdot \mathbf{d}_{j, s}+\left.\mathbf{d}_{k} \cdot \delta \mathbf{d}_{j, s}\right|_{t=0}\right]
\end{aligned}
$$


It follows, that the internal virtual work can be computed from

$$
G^{\text {int }}(\mathbf{x}, \delta \mathbf{x})=\int_{0}^{L} \delta \Gamma \cdot \mathbf{D}_{\mathbf{1}} \cdot \Gamma+\delta \mathbf{K} \cdot \mathbf{D}_{\mathbf{2}} \cdot \mathbf{K} \mathrm{d} s
$$

and the virtual work of the inertia terms is given by

$$
G^{\mathrm{kin}}(\mathbf{x}, \delta \mathbf{x})=\int_{0}^{L} A_{\rho}\left(\ddot{\boldsymbol{\varphi}}(s, t)+\ddot{\mathbf{d}}_{\alpha} S_{\alpha}\right) \cdot \delta \boldsymbol{\varphi}(s, t)+\left(S^{\alpha} \ddot{\boldsymbol{\varphi}}(s, t)+M_{\rho}^{\alpha \beta} \cdot \ddot{\mathbf{d}}_{\beta}\right) \cdot \delta \mathbf{d}_{\alpha} \mathrm{d} s
$$

The external loads, external forces and moments, result in the following virtual work expression

$$
G^{\mathrm{ext}}(\mathbf{x}, \delta \mathbf{x})=\int_{0}^{L} \delta \boldsymbol{\Gamma} \cdot \overline{\mathbf{N}}_{\mathrm{ext}}+\delta \mathbf{K} \cdot \overline{\mathbf{M}}_{\mathrm{ext}} \mathrm{d} s
$$

and the variation of the constraints leads to

$$
G^{\mathrm{con}}\left(\mathbf{d}_{i}, \boldsymbol{\lambda}, \delta \mathbf{d}_{i}, \delta \boldsymbol{\lambda}\right)=\int_{0}^{L} \boldsymbol{\lambda}: \mathbf{G} \cdot \delta \mathbf{d}_{i}+\delta \boldsymbol{\lambda}: \boldsymbol{\Phi}_{\text {in }} \mathrm{d} s
$$

where

$$
\mathbf{G}_{\text {in }}=\operatorname{Grad}\left(\boldsymbol{\Phi}_{\text {in }}\right)
$$

From the principle of virtual work follows

$$
G^{\mathrm{int}}+G^{\mathrm{kin}}-G^{\mathrm{ext}}+G^{\mathrm{con}}=0
$$

\subsection{Finite Element Discretization}

The displacements and directors are discretized in a finite element sense with NURBS shape functions. In classical FEM the shape function chosen for the discretization of the Lagrange multipliers coincide with Dirac deltas associated with the nodes [5, 7]. As the NURBS shape functions are not interpolatory, this method can no longer be applied directly. Instead one can chose to discretize the Lagrange multipliers with the NURBS shape functions. In [8] it is argued that the linear theory indicates that different orders of shape functions for displacements as for directors and Lagrange multipliers should be applied. They propose to use shape functions of the order $p_{\varphi}-1$ for the directors and Lagrange multipliers, where $p_{\varphi}$ is the order of the shape functions applied to discretize the displacements. However, a proof for the optimal discretization is not given.

Under the assumption of the arbitrariness of the test functions we obtain the following timecontinuous system of differential algebraic equations (DAE's)

$$
\begin{aligned}
\mathbf{M} \ddot{\mathbf{q}}+\nabla V(\mathbf{q})+\mathbf{G}_{\text {in }}^{\top} \boldsymbol{\lambda} & =\mathbf{f}_{\mathrm{ext}} \\
\boldsymbol{\Phi}_{\text {in }}(\mathbf{q}) & =\mathbf{0}
\end{aligned}
$$

where $\mathbf{q}$ contains the values of the control points of the displacements and directors and $\boldsymbol{\lambda}$ contains the values of the Lagrange multipliers.

\subsubsection{Initialization of Directors}

In the IGA the control points, which correspond to the degrees of freedom, in general do not lie inside the physical domain. The quantities inside the physical domain are given by the linear combination of the values on the control points multiplied with the shape functions. The noninterpolatory nature of the shape functions has to be taken into account in the definition of the directors in a preprocessing step. An algorithm, where the directors in every integration point are used to compute the directors on the control points is introduced in [22]. We use the proposed algorithm. The directors of the beam at the Gauss points are given by

$$
\mathbf{d}^{\mathbf{h}}\left(s_{\mathrm{GP}}\right)=\sum_{j=1}^{n_{\mathrm{CP}}} N_{i}\left(s_{\mathrm{GP}}\right) \mathbf{d}_{i, \mathrm{CP}}^{j}
$$


where $\mathbf{d}_{i}^{h}\left(s_{\mathrm{GP}}\right)$ are the discretized directors on the Gauss points and $\mathbf{d}_{i, \mathrm{CP}}^{A}$ the directors on the control points. Note that $s$ can be used interchangeably with $\xi$. Eq. (31) can be written in matrix notation

$$
\mathbf{d}_{i}^{\mathrm{GP}}=\mathbf{N} \mathbf{d}_{i}^{\mathrm{CP}}
$$

where $\mathbf{N}$ has the dimension of $\left(n_{\mathrm{GP}} \cdot n_{e l} \times n_{\mathrm{CP}}\right) . n_{\mathrm{GP}}$ is the number of Gauss points per element and $n_{e l}$ the number of elements. By multiplying with $\mathbf{N}^{\top}$ we obtain the following normal equations

$$
\mathbf{N}^{\top} \mathbf{d}_{i}^{\mathrm{GP}}=\mathbf{N}^{\top} \mathbf{N} \mathbf{d}_{i}^{\mathrm{CP}}
$$

The matrices $\mathbf{N}^{\top} \mathbf{d}_{i}^{\mathrm{GP}}$ and $\mathbf{N}^{\top} \mathbf{N}$ can be assembled element wise. The values of the director vectors on the control points follow than from

$$
\left(\mathbf{N}^{\top} \mathbf{N}\right)^{-1} \mathbf{N}^{\top} \mathbf{d}_{i}^{\mathrm{GP}}=\mathbf{d}_{i}^{\mathrm{CP}}
$$

\subsection{Numerical Objectivity Test}

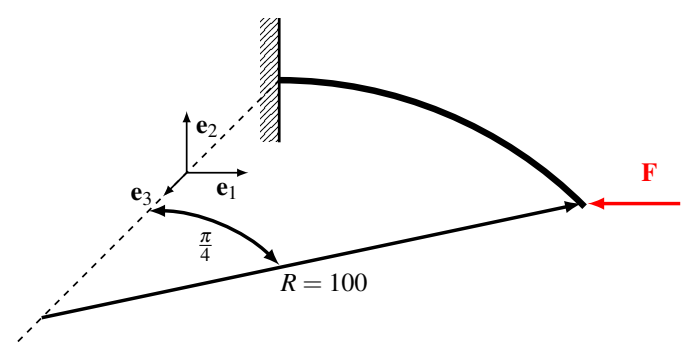

Figure 2. 3D cantilever bend

Geometrically exact beam formulations based on a discretization of rotations may suffer from a lack of objectivity, if the discretization is not performed with great care [4]. To show that the presented formulation does not suffer from this problem the following example from [5] is simulated. A 3D cantilever bend as shown in Fig. 2 is investigated. The stiffness properties of the beam are $G A_{1}=G A_{2}=5 \cdot 10^{6}, E A=10^{7}$ and $E I_{1}=E I_{2}=G J=\frac{1}{12} \cdot 10^{7}$. As shown the cantilever is fixed at one end. On the other end of the beam the force $\mathbf{F}=\left[\begin{array}{lll}F_{1}, & F_{2}, & F_{3}\end{array}\right] \mathbf{e}_{i}$ acts. The force is applied in increments of $\left\|\Delta F_{i}\right\|=25$. In Table 1 the load cycle is shown in detail. The Newton-Raphson solver is considered converged for a Euclidean norm of $\|\mathbf{g}\| \leq \varepsilon=10^{-7}$ of the residual $\mathbf{g}$. In Table

Table 1. Load cycle and tip displacement $\boldsymbol{\varphi} \cdot \mathbf{e}_{2}$ of the 3D cantilever bend

\begin{tabular}{lccc}
\hline \multicolumn{3}{l}{ Load levels } & \multicolumn{3}{c}{ polynomial order } \\
{$\left[\begin{array}{lll}F_{1}, & F_{2}, & F_{3}\end{array}\right]$} & $p=1$ & $p=2$ & $p=3$ \\
\hline$\left[\begin{array}{lll}0 & 0 & 0\end{array}\right]$ & 0 & 0 & 0 \\
{$\left[\begin{array}{lll}-600 & 0 & 0\end{array}\right]$} & 0 & 0 & 0 \\
{$\left[\begin{array}{lll}-600 & 600 & 0\end{array}\right]$} & 61.9106 & 59.9274 & 58.9219 \\
{$\left[\begin{array}{lll}-600 & 600 & 600\end{array}\right]$} & 40.1867 & 38.2493 & 37.7772 \\
{$\left[\begin{array}{lll}0 & 600 & 600\end{array}\right]$} & 37.9928 & 36.2738 & 36.7271 \\
{$\left[\begin{array}{lll}0 & 0 & 600\end{array}\right]$} & $\ll \varepsilon$ & $\ll \varepsilon$ & $\ll \varepsilon$ \\
{$\left[\begin{array}{lll}0 & 0 & 0\end{array}\right]$} & $\ll \varepsilon$ & $\ll \varepsilon$ & $\ll \varepsilon$ \\
\hline
\end{tabular}

1 the displacement of the tip in the direction of $\mathbf{e}_{2}$ is presented. After the whole load cycle is completed the configuration has to coincide with the initial configuration again. The cantilever bend is discretized with 8 beam elements. The simulations are conducted for polynomial orders from $p=1$ to $p=3$. All results are in good agreement with [5]. It can be seen that the displacement in direction of $\mathbf{e}_{2}$ is zero in a numerical sense at the end of the load cycle and, thus, coincides with the initial configuration as expected. 


\subsection{Time Discretization}

As described in Sec. 1 it is of great advantage to use an energy-momentum scheme in combination with geometrically exact beams. Using the energy-momentum scheme described in [19] the fully discretized DAE for the geometrically exact beam reads as

$$
\begin{array}{r}
\mathbf{M a}_{n+\frac{1}{2}}+\bar{\nabla} V\left(\mathbf{q}_{n}, \mathbf{q}_{n+1}\right)+\mathbf{G}_{\mathrm{in}}{ }^{\top}\left(\mathbf{q}_{n+\frac{1}{2}}\right) \boldsymbol{\lambda}_{n+1}=0 \\
\boldsymbol{\Phi}\left(\mathbf{q}_{n+1}\right)=0
\end{array}
$$

where

$$
\begin{aligned}
\mathbf{q}_{n+\frac{1}{2}} & =\frac{1}{2}\left(\mathbf{q}_{n}+\mathbf{q}_{n+1}\right) \\
\mathbf{v}_{n} & =\frac{2}{\Delta t}\left(\mathbf{q}_{n}-\mathbf{q}_{n-1}\right)-\mathbf{v}_{n-1} \\
\mathbf{a}_{n+\frac{1}{2}} & =\frac{2}{\Delta t^{2}}\left(\mathbf{q}_{n+1}-\mathbf{q}_{n}\right)-\frac{2}{\Delta t} \mathbf{v}_{n}
\end{aligned}
$$

$\Delta t$ is the time step and $\mathbf{q}_{n}$ the nodal values at time $n \Delta t$, and $\mathbf{q}_{n+1}$ the nodal values at time $(n+1) \Delta t$ respectively (analogous for $\boldsymbol{\lambda}_{\mathbf{n}+\mathbf{1}}$ ). $\bar{\nabla} \mathbf{f}$ is called the discrete gradient of a function $\mathbf{f}$. According to Gonzalez [9] it has to fulfill the following properties to inherit the symmetry properties (concerning linear and angular momentum and energy) from the continuous problem

- directionality condition

$$
\bar{\nabla} \mathbf{f}\left(\mathbf{q}_{n}, \mathbf{q}_{n+1}\right)\left(\mathbf{q}_{n+1}-\mathbf{q}_{n}\right)=\mathbf{f}\left(\mathbf{q}_{n+1}\right)-\mathbf{f}\left(\mathbf{q}_{n}\right)
$$

- consistency condition

$$
\bar{\nabla} \mathbf{f}\left(\mathbf{q}_{n}, \mathbf{q}_{n+1}\right)=\bar{\nabla} \mathbf{f}\left(\frac{\mathbf{q}_{n}+\mathbf{q}_{n+1}}{2}\right)+\mathscr{O}\left(\left\|\mathbf{q}_{n+1}-\mathbf{q}_{n}\right\|\right)
$$

In case of a quadratic strain energy function the discrete gradient uses the average of the strains and not the average of the configurations such as with the midpoint rule [10].

\subsubsection{Beam with Concentrated Masses}

In Fig. 3 the initial configuration of a beam with concentrated masses is shown. This problem is taken from [6]. The beam has a length of $2 L$, where $L=1 \mathrm{~m}$. At both ends as well as in the middle of the beam concentrated masses are added with $M=10 \mathrm{~kg}$ and $m=1 \mathrm{~kg}$. The beam itself has a mass density per unit length of $A_{\rho}=0.27 \frac{\mathrm{kg}}{\mathrm{m}}$ and a mass-moment of inertia of the cross-section of $M_{\rho}=9 \cdot 10^{-8} \frac{\mathrm{kg}}{\mathrm{m}}$. The beam stiffness parameters are given as $E I=2.43 \mathrm{Nm}^{2}, G J=586 \mathrm{Nm}^{2}$, $G A=2.43 \cdot 10^{6} \mathrm{~N}$ and $E A=7.3 \cdot 10^{6} \mathrm{~N}$.

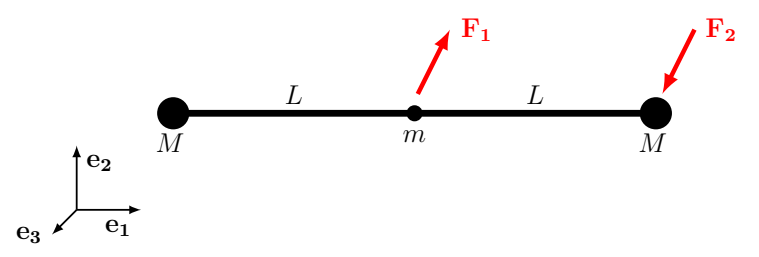

Figure 3. Initial configuration of the beam with concentrated masses.

The system is exited by two time-dependent external dead loads $\mathbf{F}_{\mathbf{1}}$ and $\mathbf{F}_{\mathbf{2}}$

$$
\mathbf{F}_{\alpha}=\mathbf{P}_{\alpha} f(t) \quad \begin{aligned}
& \mathbf{P}_{1}=-1 \mathrm{Ne}_{\mathbf{1}}-3 \mathrm{Ne}_{\mathbf{3}} \\
& \mathbf{P}_{2}=2 \mathrm{Ne}_{\mathbf{1}}+4 \mathrm{Ne}_{\mathbf{3}}
\end{aligned}
$$


The time-dependent function $f(t)$ is given by

$$
f(t)= \begin{cases}\frac{1}{2}\left(1-\cos \left(\frac{2 \pi t}{T}\right)\right) & \text { for } t \leq T \\ 0 & \text { for } t \geq T\end{cases}
$$

where $T=3 \mathrm{~s}$. The simulation results for the angular momentum $\mathbf{L}=\left[\begin{array}{lll}L_{1}, & L_{2}, & L_{3}\end{array}\right] \mathbf{e}_{i}$ and the
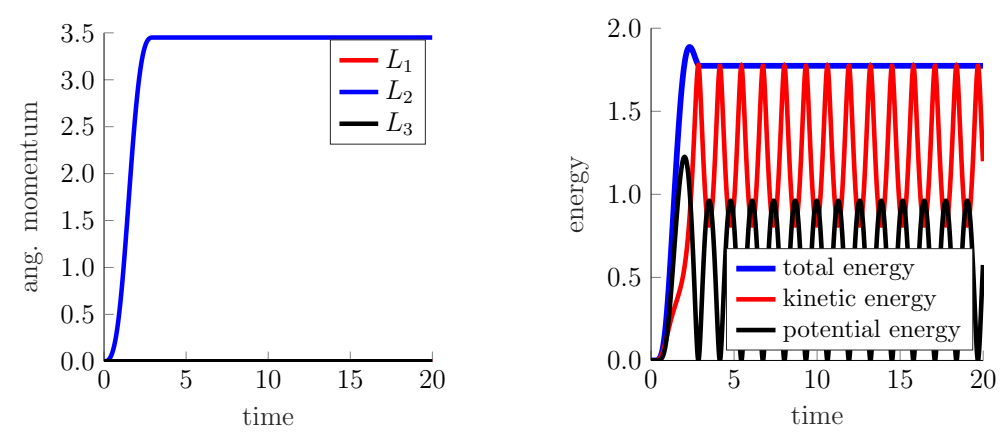

Figure 4. Angular momentum and energy over time for the beam with concentrated masses.

energy of the system are shown in Fig. 4. A constant time steps size of $\Delta t=1 \cdot 10^{-2} \mathrm{~s}$ is used and the beam is discretized with 22 linear elements. All results are in very good agreement with [6]. It can be seen very easily, that for $t>T$ the component $L_{2}$ in $\mathbf{e}_{2}$-direction of the angular momentum is constant (both other components are zero for all $t$ ). The same effect can be found when considering the total energy of the system. It is constant, after all external loads are set to zero.

\section{Sliding Joint}

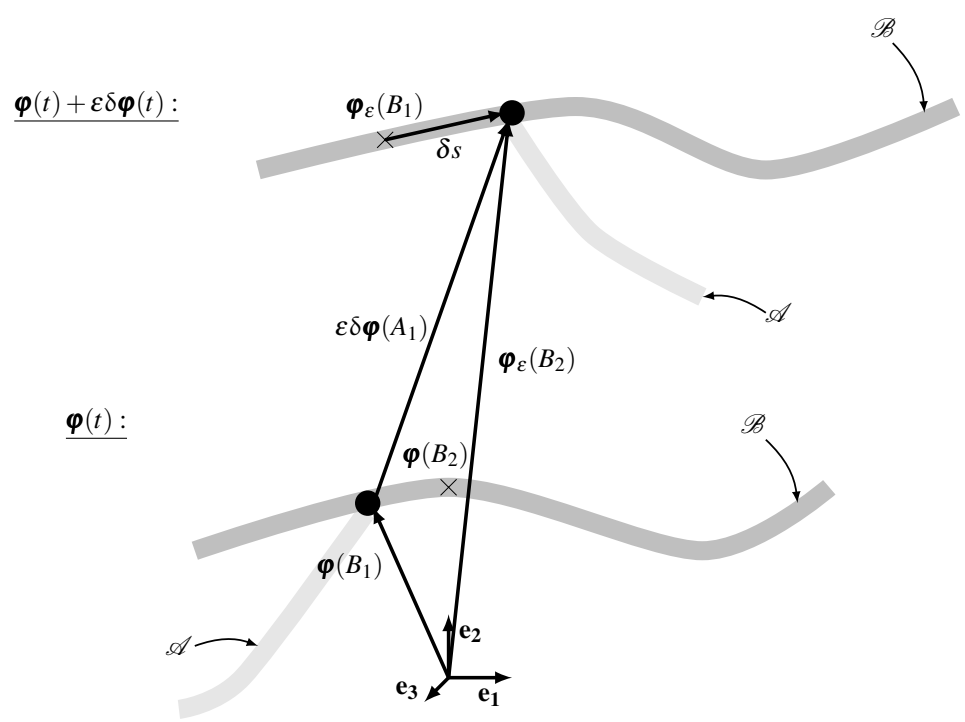

Figure 5. Sketch of two beams with sliding contact without and with pertubation.

We assume that two beams, $\mathscr{A}$ and $\mathscr{B}$, are in contact at one point in the deformed configuration $\boldsymbol{\varphi}(t)$ as shown in Fig. 5. We make the following assumptions describing the physical system

1. The material point $A_{1}$ on beam $\mathscr{A}$ is always in contact with beam $\mathscr{B}$, whereas the contact point on beam $\mathscr{B}$ may change over time. Beam $\mathscr{A}$, therefore, slides along beam $\mathscr{B}$ 
2. The assumption of a frictionless contact is made. Thus, the contact forces act only in normal direction

$$
\mathbf{N}_{I}=\mathbf{n}_{I}\left\|\mathbf{N}_{I}\right\| \quad \text { where } I=A_{1}, B_{1}
$$

where the subscribed $A$ denotes that the variable belongs to beam $\mathscr{A}$ and the subscribed $B$ denotes that the variable belongs to beam $\mathscr{B}$. From those assumptions the constraints for a spherical sliding joint follow as

$$
\boldsymbol{\Phi}_{\mathrm{ex}}\left(\mathbf{q}_{\mathbf{n}}\right)=\left[\begin{array}{l}
\left(\boldsymbol{\varphi}_{A}\left(A_{1}\right)-\boldsymbol{\varphi}_{B}\left(B_{1}\right)\right) \cdot \mathbf{n}_{1} \\
\left(\boldsymbol{\varphi}_{A}\left(A_{1}\right)-\boldsymbol{\varphi}_{B}\left(B_{1}\right)\right) \cdot \mathbf{n}_{2}
\end{array}\right]
$$

The local normal vectors $\mathbf{n}_{\alpha}$ are given by

$$
\begin{aligned}
\mathbf{t} & =\frac{\left.\boldsymbol{\varphi}_{B, s}\right|_{s=s_{\mathrm{c}}}}{\left\|\left.\boldsymbol{\varphi}_{B, s}\right|_{s=s_{\mathrm{c}}}\right\|} \\
\mathbf{n}_{1} & =\frac{\left.\boldsymbol{\varphi}_{B, s}\right|_{s=s_{\mathrm{c}}}}{\left\|\left.\boldsymbol{\varphi}_{B, s}\right|_{s=s_{\mathrm{c}}}\right\|} \\
\mathbf{n}_{2} & =\mathbf{n}_{1} \times \mathbf{t}_{3}
\end{aligned}
$$

where $s_{\mathrm{c}}$ is the arc-length of beam $\mathscr{B}$, which is closest to the point of contact of beam $\mathscr{A}$. Here the assumption is made that beam $\mathscr{A}$ is in contact at the last control point at $s_{A}=1 . s_{\mathrm{c}}$ has to be determined in each time step. An efficient algorithm for this task can be found in [21].

\subsection{Null Space Method}

A transformation of a system of DAE's to a system of ordinary differential equations (ODE's) can be achieved using the so-called null space projection [17]. A general constrained mechanical system is governed by a system of equations of the following form

$$
\begin{aligned}
\mathbf{M} \ddot{\mathbf{q}}+\nabla V(\mathbf{q})+\mathbf{G}^{\top}(\mathbf{q}) \boldsymbol{\lambda} & =0 \\
\mathbf{\Phi}(\mathbf{q}) & =0
\end{aligned}
$$

where $\mathbf{q} \in \mathbb{R}^{u}$ are the redundant coordinates and $\boldsymbol{\lambda} \in \mathbb{R}^{v}$ are the Lagrange multipliers. By multiplication with a null space matrix $\mathbf{P}$, from the null space of the gradient $\mathbf{G}$, the constraint forces $\left(\mathbf{G}^{\top}(\mathbf{q}) \boldsymbol{\lambda}\right)$ can be can be eliminated from Eq. (47), as $\mathbf{G P}=\mathbf{0}$. When additionally a mapping $\mathbf{F}(\mathbf{u})=\mathbf{q}$ from $U \in \mathbb{R}^{u-v}$ to $Q \in \mathbb{R}^{u}$ is introduced, the DEA can be rewritten into an ODE

$$
\mathbf{P}^{\top}[\mathbf{M} \ddot{\mathbf{q}}+\nabla V(\mathbf{q})]=0
$$

An approach similar to the null space projection, called master-slave approach, was developed to simulate various joints between beams $[13,14,15,16]$. Here, the null space matrix is constructed through geometrical reasoning without the introduction of constraints. The contact condition for a sliding spherical joint in a perturbed configuration (see Fig. 5) is given by

$$
\boldsymbol{\varphi}_{\varepsilon}\left(A_{1}\right)=\boldsymbol{\varphi}_{\varepsilon}\left(B_{2}\right)
$$

Thus, the virtual displacement $\delta \varphi\left(A_{1}\right)$ can be expressed as

$$
\delta \boldsymbol{\varphi}\left(A_{1}\right)=\left.\frac{\mathrm{d}}{\mathrm{d} \varepsilon}\right|_{\varepsilon=0} \boldsymbol{\varphi}_{\varepsilon}\left(A_{1}\right)=\left.\frac{\mathrm{d}}{\mathrm{d} \varepsilon}\right|_{\varepsilon=0} \boldsymbol{\varphi}_{\varepsilon}\left(B_{2}\right)=\boldsymbol{\varphi}_{, s}\left(B_{1}\right) \delta s+\delta \boldsymbol{\varphi}\left(B_{1}\right)
$$

where $\delta s$ is the variation of the arc-length. After discretizing the displacements and directors in a finite element sense, the virtual displacements can be written as

$$
\delta \mathbf{q}_{n}=\mathbf{P}_{\mathrm{ex}} \delta \mathbf{q}_{\mathbf{h}}
$$


where the virtual displacements $\delta \mathbf{q}_{\mathbf{h}} \in \mathbb{R}^{12(a+b)-2}$ are given by

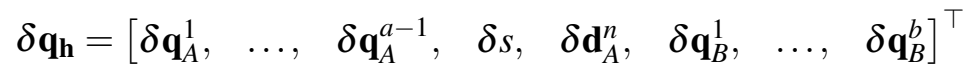

$a$ is the number of control points of beam $\mathscr{A}$, respectively, $b$ is the number of control points of beam $\mathscr{B}$. The null space matrix $\mathbf{P}_{\mathrm{ex}} \in \mathbb{R}^{(12(a+b)) \times(12(a+b)-2)}$ is thus given by

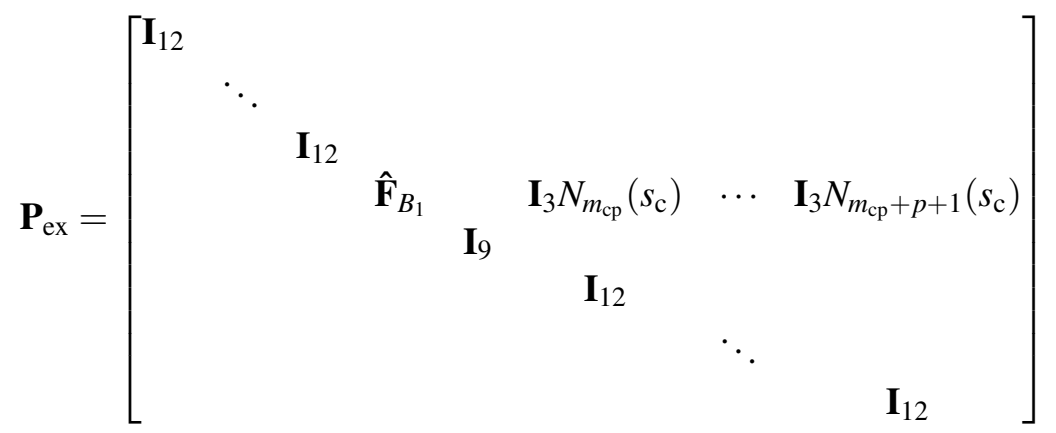

where

$$
\hat{\mathbf{F}}_{B_{1}}=\sum_{i=m_{\mathrm{cp}}}^{m_{\mathrm{cp}}+p+1} N_{i, s}\left(s_{\mathrm{c}}\right) \boldsymbol{\varphi}^{i}
$$

and $\mathbf{I}_{12} \in \mathbb{R}^{12 \times 12}$ is the identity matrix $\left(\mathbf{I}_{9} \in \mathbb{R}^{9 \times 9}\right.$ and $\mathbf{I}_{3} \in \mathbb{R}^{3 \times 3}$, respectively). The index $i$ runs from $m_{\mathrm{cp}}$ to $m_{\mathrm{cp}+p+1}$, thus including all relevant shape functions and control points of beam $\mathscr{B}$ to display the displacement $\boldsymbol{\varphi}_{B}\left(B_{1}\right)$ at $s_{\mathrm{c}}$.

The reparametrization of the sliding joint contact is given by

$$
\mathbf{F}(\mathbf{u})=\left[\begin{array}{c}
\mathbf{q}_{n A}^{1}+\mathbf{u}_{A}^{1} \\
\vdots \\
\mathbf{q}_{n A}^{n-1}+\mathbf{u}_{A}^{a-1} \\
\sum_{i=m_{\mathrm{cp}}}^{m_{\mathrm{cp}}+p+1} N_{i}\left(s_{\mathrm{c} n+1}\right)\left(\boldsymbol{\varphi}_{n B}^{i}+\mathbf{u}_{\varphi_{B}}^{i}\right) \\
\mathbf{d}_{n A}^{n}+\mathbf{u}_{\mathbf{d}} A^{a} \\
\mathbf{q}_{n B}^{1}+\mathbf{u}_{B}^{1} \\
\vdots \\
\mathbf{q}_{n B}^{m}+\mathbf{u}_{B}^{b}
\end{array}\right]
$$

where $\mathbf{u}_{\square \bigcirc}^{(\bullet)}$ is the incremental displacement of control point $(\bullet)$, variable $\square$ and beam $\bigcirc$.

So far the null space matrix $\mathbf{P}_{\mathrm{ex}}$ is time continuous. Two conditions for the discretized null space matrix are given in [18]

1. For time steps $\Delta t \rightarrow 0$ the discrete matrix has to coincide with the continuous one

$$
\mathbf{P}\left(\mathbf{q}_{n}, \mathbf{q}_{n+1}\right) \rightarrow \mathbf{P}\left(\mathbf{q}_{n}\right) \quad \text { as } \quad \mathbf{q}_{n+1} \rightarrow \mathbf{q}_{n}
$$

2. It is further required that

$$
\mathbf{G}\left(\mathbf{q}_{n}, \mathbf{q}_{n+1}\right) \mathbf{P}\left(\mathbf{q}_{n}, \mathbf{q}_{n+1}\right)=\mathbf{0}
$$

Taking into account the above conditions the discrete null space matrix $\mathbf{P}_{\mathrm{ex}}\left(\mathbf{q}_{n}, \mathbf{q}_{n+1}\right)$ pertaining to the (external) sliding constraints can now be devised as discrete version of (53).

Note that the internal constraints associated with the orthonormality of the directors still need to be taken into account to complete the discrete null space method. To this end, an additional null space matrix $\mathbf{P}_{\text {in }}$ can be applied. Furthermore, the reparametrization $\mathbf{F}(\mathbf{u})$ needs be adjusted appropriately. These additional steps required to complete the discrete null space method can be found in [19] for the geometrically exact beam based on a director formulation. 


\section{Conclusion / Outlook}

A short introduction of the used NURBS shape functions is given in Sec. 2. The shape functions are implemented into the well-known geometrically exact beam theory. A description based on directors is hereby used as described in Sec. 3. The orthonormality of the directors is only enforced in a weak sense. The objectivity of the implemented formulation is shown in the 3D cantilever bend example. For the time discretization an energy-momentum scheme is employed. The conervation properties of the scheme are shown in the example of the beam with concentrated masses.

Two algorithms for the sliding spherical joint are introduced. Both are expected to be energy and momentum-conserving. The accuracy of both algorithms and their conserving properties has still to be proven on numerical examples.

\section{REFERENCES}

[1] Simo, J.C.: A finite strain beam formulation. The three-dimensional dynamic problem. Part I. Computer Methods in Applied Mechanics and Engineering 49(1) (1985) 55-70 doi:10.1016/0045-7825(85)90050-7.

[2] Simo, J.C., Vu-Quoc, L.: A three-dimensional finite-strain rod model. part II: Computational aspects. Computer Methods in Applied Mechanics and Engineering 58(1) (1986) 79-116 doi:10.1016/0045-7825(86)90079-4.

[3] Simo, J.C., Vu-Quoc, L.: On the dynamics of flexible beams under large overall motions-the plane case: Part I. Journal of Applied Mechanics 53(4) (1986) 849-854 doi:10.1115/1.3171871.

[4] Crisfield, M.A., Jelenic, G.: Objectivity of strain measures in the geometrically exact three-dimensional beam theory and its finite-element implementation. Proceedings of the Royal Society A Mathematical, Physical and Engineering Sciences 455 (1998) 1125-1147 doi:10.1098/rspa.1999.0352.

[5] Betsch, P., Steinmann, P.: Frame-indifferent beam finite elements based upon the geometrically exact beam theory. International Journal for Numerical Methods in Engineering 54(12) (2002) 1775-1788 doi:10.1002/nme.487.

[6] Betsch, P., Steinmann, P.: Constrained dynamics of geometrically exact beams. Computational Mechanics 31 (2003) 49-59 doi:10.1007/s00466-002-0392-1.

[7] Romero, I., Armero, F.: An objective finite element approximation of the kinematics of geometrically exact rods and its use in the formulation of an energy-momentum conserving scheme in dynamics. International Journal for Numerical Methods in Engineering 54(12) (2002) 1683-1716 doi:10.1002/nme.486.

[8] Harsch, J., Capobianco, G., Eugster, S.R.: Finite element formulations for constrained spatial nonlinear beam theories. Mathematics and Mechanics of Solids (2021) doi:10.1177/10812865211000790.

[9] Gonzalez, O.: Time Integration and Discrete Hamiltonian Systems. Journal of Nonlinear Science 6(5) (1996) 449-467 doi:10.1007/BF02440162.

[10] Simo, J.C., Tarnow, N.: The discrete energy-momentum method. Conserving algorithms for nonlinear elastodynamics. ZAMP Zeitschrift für angewandte Mathematik und Physik 43(5) (1992) 757-792 doi:10.1007/BF00913408.

[11] Simo, J.C., Tarnow, N., Doblare, M.: Non-linear dynamics of three-dimensional rods: Exact energy and momentum conserving algorithms. International Journal for Numerical Methods in Engineering 38(9) (1995) 1431-1473 doi:10.1002/nme.1620380903. 
[12] Cottrell, J.A., Hughes, T.J., Bazilevs, Y.: Isogeometric Analysis - Toward Integration of CAD and FEA. John Wiley and Sons, Ltd, West Sussex (2009) ISBN:9780470748732.

[13] Jelenić, G., Crisfield, M.A.: Non-linear 'master-slave' relationships for joints in 3-D beams with large rotations. Computer Methods in Applied Mechanics Engineering 135(3-4) (1996) 211-228 doi:10.1016/0045-7825(96)01017-1.

[14] Muñoz, J.J., Jelenić, G.: Sliding contact conditions using the master-slave approach with application on geometrically non-linear beams. International Journal of Solids and Structures 41(24-25) (2004) 6963-6992 doi:10.1016/j.ijsolstr.2004.05.032.

[15] Muñoz, J.J.: Modelling unilateral frictionless contact using the null-space method and cubic B-Spline interpolation. Computer Methods in Applied Mechanics and Engineering 197(912) (2008) 979-993 doi:10.1016/j.cma.2007.09.022.

[16] Sibilieau, A., Muñoz, J.J.: Conserving time-integration of beams under contact constrains using B-Spline interpolation. In: MULTIBODY DYNAMICS 2011, ECCOMAS Thematic Conference, Brussels, Belgium (July 2011)

[17] Betsch, P.: The discrete null space method for the energy consistent integration of constrained mechanical systems. Part I: Holonomic constraints. Computer Methods in Applied Mechanics and Engineering 194(50-52) (2005) 5159-5190 doi:10.1016/j.cma.2005.01.004.

[18] Betsch, P., Leyendecker, S.: The discrete null space method for the energy consistent integration of constrained mechanical systems. Part II: Multibody dynamics. International Journal for Numerical Methods in Engineering 67(4) (2006) 499-552 doi:10.1002/nme.1639.

[19] Leyendecker, S., Betsch, P., Steinmann, P.: The discrete null space method for the energyconsistent integration of constrained mechanical systems. Part III: Flexible multibody dynamics. Multibody System Dynamics 19(1-2) (2008) 45-72 doi:10.1007/s11044-007-90564.

[20] Hughes, T., Cottrell, J., Bazilevs, Y.: Isogeometric analysis: CAD, finite elements, NURBS, exact geometry and mesh refinement. Computer Methods in Applied Mechanics and Engineering 194(39) (2005) 4135-4195 doi:10.1016/j.cma.2004.10.008.

[21] Piegl, L., Tiller, W.: The NURBS Book. Springer-Verlag Berlin Heidelberg GmbH, Berlin (1997) ISBN:978354061545-3.

[22] Dornisch, W., Klinkel, S., Simeon, B.: Isogeometric Reissner-Mindlin shell analysis with exactly calculated director vectors. Computer Methods in Applied Mechanics and Engineering 253 (2013) 491-504 doi:10.1016/j.cma.2012.09.010. 